\title{
Factores asociados con la búsqueda del servicio de interrupción legal del embarazo en la Ciudad de México, 2010
}

\author{
Alejandro Figueroa-Lara, M en C,(1) Belkis Aracena-Genao, M en C, , $^{(2)}$ \\ Hortensia Reyes-Morales, PhD, (2) Héctor Lamadrid-Figueroa, PhD. (2)
}

\author{
Figueroa-Lara A, Aracena-Genao B, \\ Reyes-Morales $\mathrm{H}$, Lamadrid-Figueroa $\mathrm{H}$. \\ Factores asociados con la búsqueda \\ del servicio de interrupción legal \\ del embarazo en la Ciudad de México, 2010. \\ Salud Publica Mex 2012;54:40I-409.
}

\section{Resumen}

Objetivo. Identificar factores asociados con la búsqueda del servicio de interrupción legal del embarazo (ILE) en la Ciudad de México. Material y métodos. Se utilizó un diseño casoscontroles. Usuarias del servicio de ILE fueron definidas como casos, y usuarias de control prenatal con 13 o más semanas de gestación con un embarazo no deseado constituyeron los controles. Se ajustaron modelos de regresión logística condicional. Resultados. Los años de escolaridad ( $R M=1.47$, IC:I.04-2.07), la ocupación (estudiante, RM=7.3 I, IC: I.5833.95; tener empleo remunerado, RM= I3.43, IC:2.04-88.54) y número de interrupciones de embarazo previas (RM=II.4I, IC:I.65-79.07) se asociaron con la búsqueda de ILE. El factor de mayor peso fue la ocupación; las mujeres que trabajan tuvieron I3.4 veces mayor posibilidad de demandar el servicio de ILE. Conclusiones. En el contexto de la Ciudad de México, mujeres con más educación y participación laboral activa utilizan más los servicios de ILE. Se requieren estrategias dirigidas a incrementar el uso de estos servicios por mujeres menos favorecidas.

Palabras clave: aborto legal/utilización; embarazo no deseado; México
Figueroa-Lara A, Aracena-Genao B, Reyes-Morales $\mathrm{H}$, Lamadrid-Figueroa $\mathrm{H}$.

Factors associated with the seeking

of legal induced abortion

services in Mexico City in 2010.

Salud Publica Mex 20 I2;54:40 I-409.

\section{Abstract}

Objective. To identify factors associated with the seeking of the legal-interruption-pregnancy (LIP) services in Mexico City. Materials and methods. We used a case-control design. Users who utilized the LIP were defined as cases, while users of the antenatal care service with gestational age 13 or more weeks and who reported having an unwanted pregnancy were defined as controls. Logistic regressions were fitted to estimate odds ratios. Results. Higher level of education (OR=I.47, 95\% Cl:I.04-2.07), women's occupation (being student OR=7.3I, 95\% Cl:I.58-33.95; worker $\mathrm{OR}=13.43,95 \% \mathrm{Cl}: 2.04-88.54)$, and number of previous abortions (OR=II.4I, 95\% Cl:I.65-79.07) were identified as factors associated with the lookup of LIP. Conclusions. In Mexico City context, empowered women with a higher level of education, or having a work activity are the users of LIP services. Strategies for improving access of women with low empowerment conditions are needed.

Key words: abortion, legal/utilization; pregnancy, unwanted; Mexico

(I) Salud, Población y Economía, AC. México DF, México.

(2) Instituto Nacional de Salud Pública. Cuernavaca, Morelos, México.

Fecha de recibido: I de julio de 20II • Fecha de aceptado: I4 de febrero de 2012 Autor de correspondencia: Belkis Aracena. Instituto Nacional de Salud Pública. Av. Universidad 655, col. Santa María Ahuacatitlán. 62100, Cuernavaca, Morelos, México.

Correo electrónico: belkis.aracena@insp.mx 
$\mathrm{A}^{\mathrm{n}}$ nivel mundial, se realizan alrededor de 22 millones de abortos en condiciones inseguras. ${ }^{1}$ Es decir, son realizados por personas sin el entrenamiento necesario y en un ambiente carente de un estándar médico mínimo. ${ }^{2} \mathrm{El}$ aborto es responsable de $13 \%$ de la mortalidad materna en el mundo, equivalente a 47000 muertes de mujeres al año. ${ }^{3}$ En América Latina y el Caribe, se estima que cada año ocurren poco más de 4 millones de abortos inseguros, lo que causa $12 \%$ de las muertes maternas de la región. ${ }^{1}$

En México, se estimó que a principios de la década de los noventa se realizaban anualmente 500000 abortos inseguros. ${ }^{4}$ Entre 1995 y 1997, 8\% de las muertes maternas se asociaron con el aborto. ${ }^{5}$ Sin embargo, estas cifras podrían no reflejar la relación real entre mortalidad materna y la práctica de abortos, pues de acuerdo con las autoridades sanitarias el subregistro existente impide visualizar la verdadera magnitud de esta problemática. ${ }^{6}$

En abril de 2007, mediante reforma del Código Penal, se despenalizó totalmente en el Distrito Federal (DF) la interrupción del embarazo hasta la semana 12 de gestación, ${ }^{7}$ adquiriendo la mujer el derecho a recibir esta atención por el solo hecho de solicitarla. Las instituciones públicas del gobierno del Distrito Federal tienen la responsabilidad de atender las solicitudes de interrupción de embarazo aunque las solicitantes cuenten con derecho a otros servicios de salud. ${ }^{7}$ Para garantizar la atención oportuna de las solicitudes la ley especifica tiempos de prestación del servicio; así, a las solicitantes con 12 semanas de gestación se les deberá proveer el servicio en un máximo de 48 horas a partir de la solicitud, y si está por debajo de las 12 semanas, la prestación del servicio se puede extender hasta por diez días a partir de la solicitud. Por otro lado, la ley prevé que únicamente los cirujanos capacitados en el procedimiento de interrupción legal del embarazo (ILE) y los ginecoobstetras están autorizados a realizarlo. ${ }^{6}$

Se estimó entre 24000 y $137000^{8,9}$ usuarias potenciales del servicio de ILE anualmente, sin embargo, el número de mujeres que utilizaron este servicio, desde la promulgación de la ley hasta el 16 de abril de 2010, fue poco más de 39 000, es decir, un promedio de 13000 atenciones anuales, aproximadamente, ${ }^{10}$ lo que equivale a una diferencia entre el número de procedimientos estimados y registrados de entre 11000 y 124000.

La despenalización de la interrupción del embarazo no garantiza por sí sola el acceso a este tipo de servicios. La literatura muestra que las barreras de acceso a servicios de interrupción de embarazo se relacionan, tanto con la organización de los servicios de salud ${ }^{11}$ como con factores propios de las mujeres tales como escolaridad, nivel de ingreso, creencias y opiniones sobre el aborto, información sobre los requisitos para solicitar el servicio y posturas religiosas. ${ }^{12-17}$

En la Ciudad de México se desconoce cuáles elementos condicionan la búsqueda del servicio de ILE. El objetivo del presente estudio fue identificar los factores propios de las mujeres asociados con la búsqueda del servicio de ILE en la Ciudad de México en 2010. La hipótesis planteada fue que la educación, capacidad de pago, opinión sobre el aborto, ocupación y religión son factores relacionados con la búsqueda de este servicio.

\section{Material y métodos}

\section{Diseño del estudio}

Se realizó un estudio de casos y controles, pareando un control por cada caso por edad y entidad federativa de residencia. Los casos se definieron como las residentes en la Ciudad de México o el Estado de México que utilizaron el servicio de ILE en alguna de las unidades médicas pertenecientes a la Secretaría de Salud del Gobierno del Distrito Federal (SSGDF). Se reclutó una muestra definida de controles compuesta por mujeres residentes en la Ciudad de México o cualquier municipio perteneciente al Estado de México, que utilizaron el servicio de control prenatal en alguna de las unidades médicas pertenecientes a la SSGDF, con edad gestacional igual o mayor a 13 semanas, que manifestaron presentar un embarazo no deseado y que no buscaron el servicio de ILE. ${ }^{*}$ Para identificar los controles se les preguntó: ¿actualmente presenta un embarazo no deseado?

\section{Instrumento}

Todas las mujeres contestaron de manera anónima un cuestionario estructurado y piloteado previamente. Los datos fueron recogidos entre los meses de abril y mayo de 2010 en 13 hospitales y un centro de salud pertenecientes a la SSGDF. El cuestionario solicitaba información sobre edad, escolaridad, ingreso mensual, estado civil, tiempo de convivencia con la pareja, religión, ocupación y derecho al servicio médico. Además, se solicitó información sobre la historia sexual y reproductiva de la mujer y opinión acerca de los derechos reproductivos de la mujer. Las usuarias contestaron el cuestionario post-procedimiento. Las preguntas utilizadas en el cuestionario se muestran en el cuadro I.

\footnotetext{
* Es oportuno mencionar que de acuerdo con los "Lineamientos de organización y operación de los servicios de salud para la interrupción legal del embarazo", el programa no contempla la realización de abortos, las interrupciones sólo pueden realizarse hasta la décimasegunda semana de gestación.
} 
Todas las mujeres fueron informadas de que su participación en el estudio era voluntaria y que no habría consecuencias en la atención que recibirían por parte de las unidades médicas si decidían no participar. Se les enfatizó que toda la información sería tratada de manera confidencial. Se obtuvo consentimiento informado, firmado post-procedimiento, de todas las participantes. Los datos analizados forman parte de un estudio que contó con la aprobación de las comisiones de investigación y ética del Instituto Nacional de Salud Pública y de la Subcomisión de Investigación y Bioética de la SSGDF.

\section{Análisis conceptual de la información}

El modelo conductual de Andersen establece que el acceso a los servicios de salud se divide en dos etapas: búsqueda y utilización de los servicios de salud. La búsqueda es resultado de la interacción de tres tipos de variables: predisponentes, capacitantes y de necesidad. Las variables predisponentes son aquellas condiciones de los usuarios que los hacen propensos a la búsqueda del servicio; están conformadas por factores sociodemo- gráficos asociados a las actitudes y creencias en torno a la salud y la enfermedad, tales como edad, número de hijos, escolaridad, ocupación y valores sociales. Las variables capacitantes son los medios disponibles de las personas que facilitan la búsqueda del servicio; algunos de ellos son el nivel de ingreso de la persona y su condición de aseguramiento. Las variables de necesidad son todas aquellas condiciones de salud que las personas consideran que precisan atención médica. ${ }^{18}$ Para explicar el proceso de búsqueda del servicio de ILE en la Ciudad de México, se utilizó el modelo de Andersen como marco conceptual.

Para hacer comparables a las usuarias y no usuarias de ILE, se asumió que las mujeres con embarazo no deseado también percibían la interrupción de su embarazo como una necesidad; la clasificación de los factores según el tipo de variable, bajo el modelo de Andersen, se presenta en el cuadro I.

\section{Análisis estadístico de la información}

Se realizó un análisis estadístico bivariado mediante la prueba de $\mathrm{ji}^{2} \mathrm{o}$ exacta de Fisher para explorar la dife-

Cuadro I

Operacionalización de los factores, bajo el modelo conductual de Andersen

\begin{tabular}{|c|c|c|}
\hline Pregunta & Factor & Tipo de variable \\
\hline ¿Cuántos años cumplidos tiene? & Edad & Predisponente \\
\hline ¿Cuál es su estado civil actual? & Estado civil & Predisponente \\
\hline ¿Su pareja actual vive con usted todo el tiempo? & Convivencia con la pareja & Predisponente \\
\hline \multicolumn{3}{|l|}{ ¿Cuál es su religión? } \\
\hline ¿Con qué frecuencia asiste usted a los servicios religiosos? & Religión & Predisponente \\
\hline \multicolumn{3}{|l|}{ ¿Se considera usted una persona religiosa? } \\
\hline ¿A qué se dedicó usted principalmente durante la semana pasada? & Ocupación & Predisponente \\
\hline ¿Cuántos hijos tiene? & Número de hijos & Predisponente \\
\hline \multicolumn{3}{|l|}{ ¿A qué edad tuvo su primera relación sexual? } \\
\hline ¿Cuántas parejas sexuales ha tenido en el último año? & Historia sexual y reproductiva & Predisponente \\
\hline \multicolumn{3}{|l|}{ ¿Cuántos abortos previos ha experimentado? } \\
\hline \multicolumn{3}{|l|}{ En su opinión, ¿la mujer tiene derecho a decidir cuántos hijos tener } \\
\hline \multicolumn{3}{|l|}{ y en qué momento? } \\
\hline En su opinión, ¿cómo piensa que debe ser la actitud de una comunidad & Opinión sobre los derechos & Predisponente \\
\hline \multicolumn{3}{|l|}{ hacia las mujeres que han solicitado una ILE? } \\
\hline \multicolumn{3}{|l|}{ En su opinión, ¿quién debe decidir si la mujer se realiza una ILE? } \\
\hline ¿Cuántos años de estudio ha realizado? & Escolaridad & Capacitante \\
\hline En total al mes ¿cuánto es el ingreso familiar en promedio? & Ingreso mensual & Capacitante \\
\hline \multicolumn{3}{|l|}{ En total al mes ¿cuánto aporta usted al ingreso familiar? } \\
\hline ¿Tiene derecho al servicio médico? & Condición de aseguramiento & Capacitante \\
\hline
\end{tabular}

Fuente: Elaboración propia con base en las preguntas de la "Encuesta para identificar la demanda por servicios de atención del servicio de interrupción legal del embarazo en hospitales públicos de la Ciudad de México, 2010"

ILE= interrupción legal del embarazo 
rencia en la distribución de las características entre las usuarias y las no usuarias. Los factores se identificaron mediante la estimación de razones de momios (RM), calculados a través de una regresión logística condicional. La asociación es presentada como RM con un intervalo de confianza de $95 \%$. Se corrigieron problemas de colinealidad y la bondad de ajuste del modelo fue evaluada mediante el procedimiento de Hosmer y Lemeshow. ${ }^{19}$ Dos observaciones fueron excluidas del modelo final por su alta influencia en los resultados y porque ocasionaban problemas de ajuste. Los datos fueron analizados en el programa estadístico Stata 10.1 para Windows.

\section{Resultados}

Un total de 148 mujeres fueron incluidas en el estudio: 74 usuarias de ILE y 74 no usuarias. Los factores predisponentes: estado civil y ocupación resultaron estadísticamente diferentes $(p<0.05)$ entre los grupos. Lo mismo ocurre con los factores capacitantes: escolaridad e ingreso personal. El 27\% de las usuarias declararon una escolaridad de por lo menos 13 años, mientras que el porcentaje de no usuarias que declararon este nivel escolar es poco mayor a $5 \%$. Referente al ingreso personal, 62.2\% de la usuarias del servicio de ILE declararon no percibir ingreso y $79.7 \%$ de las no usuarias tampoco recibe ingresos personales. Cerca de $42 \%$ de las usuarias son mujeres solteras con pareja, entretanto, poco más de $55 \%$ de las no usuarias son mujeres que viven en unión libre. ${ }^{*}$ Respecto a la ocupación, la mayoría de las usuarias tiene un empleo remunerado o son estudiantes (33.8 y 32.4\%, respectivamente), mientras que poco más de tres cuartas partes $(75.7 \%)$ de las no usuarias son amas de casa (cuadro II).

Todos los factores predisponentes excepto el relacionado con la actitud que debe tener la comunidad hacia las mujeres usuarias del servicio de ILE mostraron una diferencia no significativa. Cerca de $34 \%$ de las usuarias opinaron que la comunidad debe tener una actitud neutral (es decir, una opinión que no apoye ni recrimine) hacia las mujeres usuarias, mientras que poco más del $13 \%$ de las no usuarias tienen esta misma opinión (cuadro III).

En el cuadro IV se muestran los resultados de la regresión logística condicional para la identificación de los factores de las mujeres asociados con la búsqueda del servicio de ILE en la Ciudad de México; debido a problemas de colinealidad, los factores sobre la frecuencia de asistencia a servicios religiosos y considerarse una per-

\footnotetext{
* Se refiere a mujeres que ya viven con su pareja, sin estar casadas ya sea por autoridades civiles o religiosas
}

sona religiosa no pudieron ser incluidos en el modelo. Un factor capacitante y dos predisponentes se asociaron significativamente con la búsqueda del servicio de ILE: los años de escolaridad (RM=1.47, IC: 1.04-2.07), la ocupación (estudiante RM=7.31, IC: 1.58 -33.95; tener empleo remunerado $\mathrm{RM}=13.43$, IC: $2.04-88.54$ ) y el número de interrupciones de embarazo previas ( $\mathrm{RM}=11.41$, IC: 1.65-79.07). De los factores mencionados anteriormente, el de mayor peso es la ocupación, pues las mujeres con un empleo remunerado tienen aproximadamente 13.4 veces mayor posibilidad de demandar el servicio con respecto a las mujeres que son amas de casa.

\section{Discusión}

Los resultados de esta investigación muestran que la búsqueda del servicio de interrupción legal del embarazo provisto por la SSGDF en la Ciudad de México, está asociada con la ocupación, número de interrupciones de embarazos previas y escolaridad.

A diferencia de los resultados de la presente investigación, los estudios de Simonds y colaboradores ${ }^{12}$ y Harden y colaboradores ${ }^{13}$ realizados en Estados Unidos y Reino Unido, respectivamente, donde la interrupción del embarazo está totalmente despenalizada, concluyeron que la filiación religiosa condiciona la búsqueda de este servicio. Cabe señalar que dentro de la literatura científica se ha mencionado que existe una diferencia entre declarar una filiación religiosa y vivir en base a los cánones de la religión, ${ }^{20}$ lo que pudiera explicar la no asociación entre la filiación religiosa declarada por las entrevistadas y la demanda por servicios de ILE provistos por la SSGDF. Por otro lado, los resultados del estudio realizado en el Reino Unido por Lee y colaboradores ${ }^{21}$ muestran que la filiación religiosa no condicionó la demanda del servicio de interrupción del embarazo de adolescentes, lo que coincide con los resultados de la presente investigación.

El estudio realizado en Suecia por Larsson y colaboradores, ${ }^{14}$ y el llevado a cabo en mujeres inmigrantes en Australia por Liamputtong, ${ }^{15}$ países con un esquema de total despenalización, concluyeron que el nivel de ingreso de las mujeres es un factor importante para la demanda del servicio de interrupción del embarazo, en contraste con los resultados de la presente investigación. La falta de asociación entre el ingreso y la búsqueda de servicios de ILE podría atribuirse a que el precio del servicio de ILE ofrecido por las unidades médicas de la SSGDF está en función del nivel de ingresos de la mujer, por lo que el precio máximo del procedimiento es de 1500 pesos. ${ }^{22}$ Los resultados obtenidos en Dinamarca por Rash y colaboradores, donde la interrupción del embarazo está totalmente 
Cuadro II

Características sociodemográficas de las mujeres encuestadas. Factores asociados con la búsQUeda del Servicio de interrupción legal del embarazo en la Ciudad de México, 2010

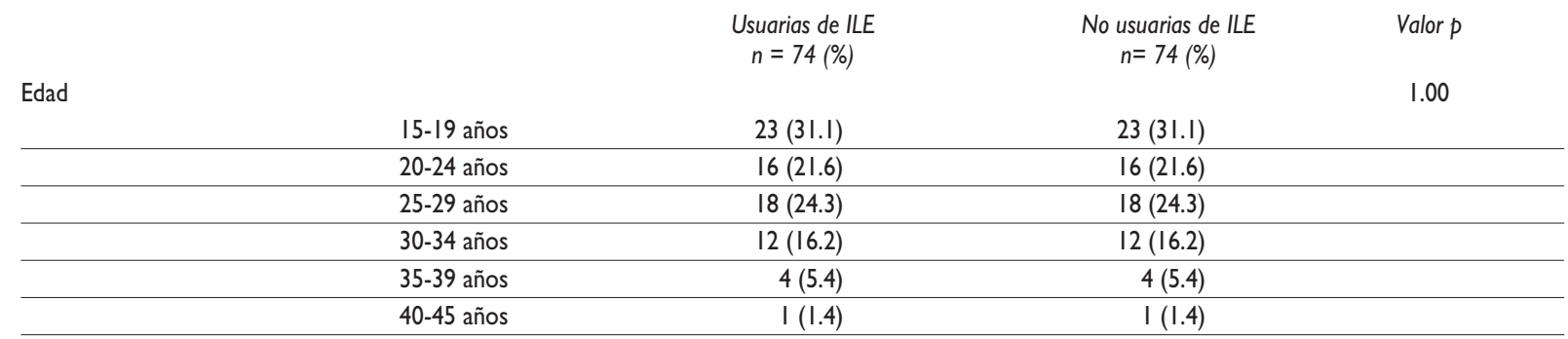

Escolaridad

\begin{tabular}{rrr}
$\leq 9$ años & $3 \mid(4 I .9)$ & $48(64.9)$ \\
\hline $10-12$ años & $23(3 \mid . I)$ & $22(29.7)$ \\
$\geq 13$ años & $20(27)$ & $4(5.4)$
\end{tabular}

Ingreso familiar mensual

$\begin{array}{rrr}\leq 2000 & 22(29.7) & 32(43.2) \\ 200 \mathrm{I}-5000 \text { pesos } & 3 \mathrm{I}(41.9) & 27(36.5) \\ 500 \mathrm{I}-9000 \text { pesos } & 8(10.8) & 6(8.1) \\ \geq 900 \mathrm{I} & 4(5.4) & 0(0) \\ \text { No contestó } & 9(12.2) & 9(12.2)\end{array}$

$\begin{array}{r}2001-5000 \text { pesos } \\ \hline 5001-9000 \text { pesos } \\ \geq 9001 \\ \hline \text { No contestó }\end{array}$

Ingreso personal mensual

$\begin{array}{rrr}0 \text { pesos } & 46(62.2) & 59(79.7) \\ \geq 1 \text { pesos } & 26(35.1) & 15(20.3) \\ \text { No contestó } & 2(2.7) & 0(0)\end{array}$

Estado civil

\begin{tabular}{rrr} 
Casada & $8(10.8)$ & $13(17.6)$ \\
\hline Soltera, con pareja & $31(41.9)$ & $5(6.8)$ \\
\hline Soltera, sin pareja & $7(9.5)$ & $12(16.2)$ \\
\hline Unión libre & $25(33.8)$ & $4 \mathrm{I}(55.4)$ \\
Otro & $3(4.1)$ & $3(4.1)$
\end{tabular}

\begin{tabular}{r} 
Religión \\
Católica \\
\hline Cristiana \\
\hline Sin religión \\
Otros
\end{tabular}

\begin{tabular}{rr} 
Casada & $8(10.8)$ \\
\hline Soltera, con pareja & $31(41.9)$ \\
\hline Soltera, sin pareja & $7(9.5)$ \\
\hline Unión libre & $25(33.8)$ \\
\hline Otro & $3(4)$.
\end{tabular}

\begin{tabular}{r} 
Ocupación \\
Ama de casa \\
\hline Estudiante \\
\hline Empleo remunerado \\
Otros
\end{tabular}

Otros

\begin{tabular}{r}
$61(82.4)$ \\
$4(5.4)$ \\
\hline $6(8.1)$ \\
$3(4.1)$
\end{tabular}

\begin{tabular}{r}
$57(77)$ \\
\hline $4(5.4)$ \\
\hline $7(9.5)$ \\
$6(8.1)$
\end{tabular}

0.772

0.033

Número de hijos

\begin{tabular}{rrr}
0 & $34(45.9)$ & $35(47.3)$ \\
\hline 1 & $18(24.3)$ & $19(25.7)$ \\
\hline 2 & $16(21.6)$ & $9(12.2)$ \\
\hline$\geq 3$ & $6(8.1)$ & $11(14.9)$
\end{tabular}

Fuente: Elaboración propia con datos de la "Encuesta para identificar la demanda por servicios de atención del servicio de interrupción legal del embarazo en hospitales públicos de la Ciudad de México, 2010" 


\section{Cuadro III}

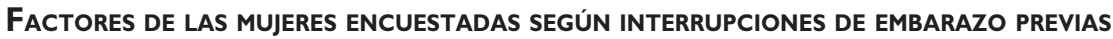

Y OPINIÓN DE LOS DERECHOS REPRODUCTIVOS DE LA MUJER

$\begin{array}{rrr}\begin{array}{r}\text { Usuarias } \\ \text { de ILE }\end{array} & \text { No usuarias } & \text { Velor } p \\ n=74(\%) & n=74(\%)\end{array}$

Número de interrupciones de embarazo previas

\begin{tabular}{rrrrr} 
& 0 & $56(75.7)$ & $64(86.5)$ \\
\hline & 1 & $15(20.3)$ & $10(13.5)$ \\
\hline
\end{tabular}

¿La mujer tiene el derecho de decidir cuántos hijos tener y en qué momento?

\begin{tabular}{rrr} 
De acuerdo & $70(94.6)$ & $62(83.8)$ \\
\hline Más o menos de acuerdo & $4(5.4)$ & $4(5.4)$ \\
\hline En desacuerdo & $0(0)$ & $1(1.4)$ \\
\hline No contestó & $0(0)$ & $7(9.5)$ \\
\hline
\end{tabular}

¿Cómo debe de ser la actitud de la comunidad hacia las mujeres que han solicitado una ILE?

\begin{tabular}{rrrr} 
Apoyo & $46(62.2)$ & $50(67.6)$ \\
\hline Neutral & $25(33.8)$ & $10(13.5)$ \\
\hline Recriminación & $3(4.1)$ & $3(4.1)$ \\
\hline
\end{tabular}

¿Quién debe decidir si la mujer se realiza una ILE?

0.057

\begin{tabular}{rrrr}
\hline La misma mujer & $32(43.2)$ & $33(44.6)$ \\
\hline La mujer y su pareja & $40(54.1)$ & $29(39.2)$ \\
\hline La pareja de la mujer & $2(2.7)$ & I (I.4) \\
\hline Otro & $0(0)$ & $5(6.8)$ \\
\hline No contestó & $0(0)$ & $6(8.1)$ \\
\hline
\end{tabular}

Fuente: Elaboración propia con datos de la "Encuesta para Identificar la demanda por servicios de atención del servicio de interrupción legal del embarazo en hospitales públicos de la Ciudad de México, 2010"

ILE= interrupción legal del embarazo

despenalizada, muestran que el nivel de ingresos no se asocia con la demanda del servicio de interrupción de embarazo. ${ }^{23}$

Respecto a la historia de interrupciones de embarazos, aunque no es posible discernir si éstas fueron legales o clandestinas, se esperaría que haber experimentado una interrupción previa aporte cierto aprendizaje o facilite el acceso a la información que se difunde sobre ILE, lo que podría explicar que el número de interrupciones previas se asocie con la búsqueda del servicio de ILE provisto por la SSGDF. Díaz y colaboradores ${ }^{24}$ también concluyeron que el número de interrupciones previas se asocia con la búsqueda del servicio.

$\mathrm{Al}$ igual que los resultados de la presente investigación, Castañeda-Abascal y colaboradores encontraron ${ }^{25}$ que tener una pareja estable no se asocia con la búsqueda del servicio de ILE. Cabezas-García y colaboradores ${ }^{26}$ concluyen que la edad de inicio de vida sexual de la mujer no se asocia con la búsqueda del servicio. En contraste con la presente investigación, la revisión sistemática por Lie y colaboradores ${ }_{,}^{27}$ con información de varios estudios realizados en Estados Unidos, reporta que la opinión sobre los derechos sexuales y la interrupción del embarazo afectan la decisión de búsqueda de dicho servicio.

La asociación de la escolaridad con la búsqueda de atención para la interrupción legal del embarazo es controversial. Los estudios realizados en Cuba por CabezasGarcía y colaboradores ${ }^{26}$ y Díaz y colaboradores, ${ }^{24}$ país donde la interrupción del embarazo está totalmente despenalizada desde finales de la década de los sesenta, 


\section{Cuadro IV}

Regresión logística condicional múltiple para la estimación de RM e IC al 95\% de los factores asociados a La interrupción legal del embarazo. Ciudad de MéXico, 2010

\begin{tabular}{|c|c|c|c|c|c|}
\hline Factores capacitantes & & $\begin{array}{c}\text { Crudo } \\
\text { RM (IC 95\%) }\end{array}$ & $\begin{array}{l}\text { Ajustado* } \\
\text { Valor } p\end{array}$ & RM (IC 95\%) & Valor $p$ \\
\hline Años de escolaridad & & NA & $1.47(1.04-2.07)$ & 0.028 & \\
\hline \multicolumn{6}{|l|}{ Ingreso familiar mensual } \\
\hline & $\leq 2000$ & 1.00 & & 1.00 & \\
\hline & $200 I-5000$ & $\mathrm{I} .67(0.52-5.3 \mathrm{I})$ & 0.385 & $0.95(0.24-3.7 I)$ & 0.941 \\
\hline & $\geq 5000$ & $3.85(0.42-35.03)$ & 0.231 & $0.59(0.04-9.69)$ & 0.712 \\
\hline
\end{tabular}

Ingreso personal mensual

$\begin{array}{lrr}0 \text { pesos } & 1.00 & 1.00\end{array}$

\begin{tabular}{lllll}
$\geq 1$ pesos & $0.94(0.18-4.99)$ & 0.940 & $0.67(0.13-3.7 I)$ & 0.649 \\
\hline
\end{tabular}

Derecho al servicio médico

No tiene aseguramiento médico

1.00

1.00

Tiene derecho a aseguramiento médico

$0.31(0.06-1.59)$

$0.162 \quad 0.27(0.05-1.45)$

0.127

Factores predisponentes

Pareja estable

\begin{tabular}{rcccc} 
Sí & 1.00 & \multicolumn{3}{c}{1.00} \\
No & $0.31(0.06-1.56)$ & 0.157 & $0.29(0.06-1.49)$ & 0.139 \\
\hline
\end{tabular}

Religión

Tiene alguna religión

1.00

1.00

No tiene religión

$0.68(0.09-4.91)$

0.704

$0.45(0.04-5.07)$

0.521

Ocupación

\begin{tabular}{|c|c|c|c|c|c|}
\hline & Ama de casa & 1.00 & \multicolumn{3}{|c|}{1.00} \\
\hline & Estudiante & $9.56(2.25-40.62)$ & $<0.01$ & $7.31(1.58-33.95)$ & $<0.01$ \\
\hline & Empleo remunerado & II.65 (2.05-66.36) & $<0.01$ & I3.43 (2.04-88.54) & $<0.01$ \\
\hline Número de hijos & & I.23 (0.65-2.33) & 0.517 & I.3I (0.67-2.58) & 0.431 \\
\hline Edad en la primera relación sexual & & I.I7 (0.84-I.62) & 0.336 & $1.09(0.75-1.59)$ & 0.632 \\
\hline Número de parejas sexuales en el último año & & $0.43(0.17-1.09)$ & 0.077 & $0.37(0.12-1.07)$ & 0.068 \\
\hline Número de interrupciones de embarazo previas & & $4.05(0.92-17.89)$ & 0.065 & II.4I (I.65-79.07) & $<0.05$ \\
\hline
\end{tabular}

Opinión acerca de los derechos reproductivos de la mujer

En desacuerdo/Más o menos

1.00

1.00

De acuerdo

$2.91(0.5 \mathrm{I}-16.92)$

$0.233 \quad 3.40(0.40-28.63)$

0.260

Opinión acerca de la actitud de una comunidad hacia una mujer que solicitó la ILE

Recriminación/Neutra

1.00

1.00

$\begin{array}{lllll}\text { Apoyo } & 0.84(0.26-2.77) & 0.783 & \mathrm{I} .19(0.32-4.38) & 0.795\end{array}$

Opinión sobre quien debe decidir si se realiza la ILE

Médico/sacerdote/pareja de la mujer/la mujer y su pareja

1.00

1.00

$\begin{array}{lllll}\text { Sólo la mujer } & 0.8 I(0.29-2.19) & 0.678 & 0.60(0.20-1.79) & 0.364\end{array}$

Fuente: Elaboración propia con datos de la "Encuesta para identificar la demanda por servicios de atención del servicio de interrupción legal del embarazo en hospitales públicos de la Ciudad de México, 2010"

ILE $=$ interrupción legal del embarazo

RM = Razón de momios

IC = Intervalo de confianza

*Ajustado por años escolaridad (variable en forma continua) 
concluyeron que las mujeres con menor nivel educativo son más propensas a buscar el servicio de interrupción del embarazo. En tanto que el estudio de CastañedaAbascal y colaboradores, ${ }^{25}$ que incluyó mujeres entre 15 y 29 años, también realizado en Cuba, concluyó que el nivel educativo de la mujer no se asocia con la búsqueda de la interrupción del embarazo. Contrario a los hallazgos de los estudios anteriores, en el contexto de la Ciudad de México, la educación y la participación en la vida laboral influyeron de manera significativa en la búsqueda del servicio de ILE, lo que podría sugerir que las mujeres más empoderadas* tienen mayor acceso a pesar de las restricciones que pueda imponer la sociedad. Lo anterior pone de manifiesto la necesidad de implementar políticas públicas para que en el largo plazo se incremente el empoderamiento de las mujeres y, en consecuencia, su capacidad de decisión, y en el corto plazo para que las que viven en condiciones de bajo empoderamiento puedan acceder a este servicio cuando ellas lo consideren necesario.

La distribución de las características de las mujeres que utilizaron el servicio de ILE en la población muestreada por la presente investigación es el de una mujer con edad entre los 15 y 24 años, con educación básica; un ingreso familiar mensual entre 2000 y 5000 pesos, sin ingresos propios, soltera con pareja, de filiación católica, de ocupación estudiante o con empleo remunerado y sin hijos. La anterior información no difiere en gran medida de las características de las mujeres que utilizaron este mismo servicio entre los años 2007 y 2011 en la SSGDF, según lo publicado por la Organización de la Sociedad Civil GIRE: $53 \%$ son solteras, cerca de $48 \%$ tienen entre 18 y 24 años de edad, 30\% con nivel de educación secundaria y alrededor de $39 \%$ con preparatoria. ${ }^{28}$

Respecto a las limitaciones de la presente investigación, es necesario reconocer que no se puede asegurar que las mujeres que manifiesten tener un embarazo no deseado necesariamente tendrán la necesidad de interrumpirlo. Sin embargo, la literatura menciona que tener esta característica hace propensa a la mujer a buscar la interrupción. ${ }^{29}$

Finalmente, hasta donde se sabe, esta es la primera investigación en explorar los factores asociados con la demanda del servicio de ILE en la Ciudad de México, por lo que creemos que es una investigación sustancial

\footnotetext{
* Definida como "Mujer con crecimiento personal, consciente de sus derechos ciudadanos y con capacidad para hacer elecciones de vida estratégicas." Está definición se tomó de la siguiente referencia: Pérez-Villar M, Vázquez-García V. Familia y empoderamiento femenino: ingresos, trabajo doméstico y libertad de movimiento de mujeres chontales de Nacajuca, Tabasco. Convergencia 2009;16(50):187-218.
}

para el estudio del fenómeno de la interrupción de embarazo en México; los resultados obtenidos representan información que puede ser relevante para mejorar el servicio de ILE. Aún existe necesidad de continuar con esta línea de investigación mediante nuevos estudios que confirmen los hallazgos que aquí se encontraron.

\section{Agradecimientos}

Al Consejo Nacional de Ciencia y Tecnología por el apoyo financiero otorgado para la realización de esta investigación. Las opiniones vertidas en este documento son responsabilidad exclusiva de los autores y no reflejan la posición oficial del CONACyT, ni de ninguna institución del Gobierno de México.

Declaración de conflicto de intereses. Los autores declararon no tener conflicto de intereses.

\section{Referencias}

I. World Health Organization. Unsafe abortion. Global and regional estimates of the incidence of unsafe abortion and associated mortality in 2008. 6a. edición. Ginebra: WHO, 2011.

2. United Nations Population Division. World population prospects. The 2002 revision. New York: UN, 2002.

3. Singh S, Wulf D, Hussain R, Bankole A, Sedgh G. Abortion worldwide: a decade of uneven progress. Nueva York: Guttmacher Institute, 2009.

4. Espinoza $H$, López C. Aborto inseguro en América Latina y el Caribe: definición del problema y su prevención. Gac Med Mex 2003; 139 suppl I:9-16.

5. Consejo Nacional de Población. Cuadernos de salud reproductiva: República mexicana. Ciudad de México: CONAPO, 2000.

6. Gobierno del Distrito Federal. Gaceta oficial del Distrito Federal 2007; 75. 2010 [consultado 2012 febrero 09]. Disponible en: http://www. ordenjuridico.gob.mx/Estatal/DISTRITO\%20FEDERAL/Lineamientos/ DFLIN26.pdf

7. Gobierno del Distrito Federal. Gaceta oficial del Distrito Federal 2007; 70. [consultado 2010 diciembre 26]. Disponible en: http://www.gire.org. $\mathrm{mx} /$ publica2/GacetaGDF_Aborto260407.pdf

8. Aracena-Genao B, Wilches-Gutiérrez JL, Figueroa-Lara A, SanhuezaSmith P, Ávila-Burgos L, Sauceda AL, et al. Interrupción legal del embarazo. Recursos disponibles en la Secretaría de Salud del Distrito Federal. Cuernavaca: INSP, 2009.

9. Juarez F, Singh S, Garcia SG, Olavarrieta CD. Estimates of induced abortion in Mexico: what's changed between 1990 and 2006? Int Fam Plan Perspect 2008; 34(4): I58-168.

10. Instituto de las Mujeres del Distrito Federal. Cifras sobre la interrupción legal del embarazo [monografía en internet]. Ciudad de México: Inmujeres, 2010 [consultado 2010 diciembre 9]. Disponible en: http:// www.inmujer.df.gob.mx/wb/inmujeres/estadisticas_sobre_ile

II. Langer-Glas A. Embarazo no deseado y el aborto inseguro: su impacto sobre la salud en México. Gac Med Mex 2003;139 suppl I:3-8.

12. Simonds W, Ellertson C, Springer K, Winikoff B. Abortion, revised: participants in the U.S. clinical trials evaluate mifepristone. Soc Sci Med 1998;46(I0):I313-1323.

13. Harden A, Ogden J. Young women's experiences of arranging and having abortions. Sociol Health IIIn 1999;2I (4):426-444. 
14. Larsson M, Aneblom G, Odlind V, Tydén T. Reasons for pregnancy termination, contraceptive habits and contraceptive failure among Swedish women requesting an early pregnancy termination. Acta Obstet Gynecol Scand 2002;8I(I):64-7I.

I5. Liamputtong P. Abortion-It is for some women only! Hmong women's perceptions of abortion. Health Care for Women Int 2003;24(3):230--24I.

16. Mogilevkina I, Hellberg D, Nordstrom M, Odlind V. Factors associated with pregnancy termination in Ukrainian women. Acta Obstet Gybecol Scand 2000;79:1 | 26-1 |31.

17. Rodgers S, Downie J. Abortion: ensuring access. CMAJ 2006; 175(I): 9. 18. Andersen RM, Davidson P. Improving access to care in America. En: Andersen RM, Rice T, Kominski G, eds. Changing the U.S. health care system: key issues in health services policy and management. 3a. edición. San Francisco: Jossey-Bass, 2007: 3-31.

19. Hosmer D, Lemeshow S. Applied logistic regression. 2nd Edition. Hoboken: Wiley, 2000.

20. Worthington E, Wade N, Hight T, Ripley J, McCullough M, Berry J, et al. The religious commitment inventory-10: development, refinement and validation of a brief scale for research and counceling. J Couns Phsychol 2003;50(I):84-96.

21. Lee E, Clements S, Ingham R, Stone N. A matter of choice? Explaining national variations in teenage abortion and motherhood [monografía en internet]. York: Joseph Rowntree Foundation, 2004 [consultado 201 I enero 9]. Disponible en: http://www.jrf.org.uk/sites/files/jrf//85935/824.pdf
22. Alianza Nacional por el Derecho a Decidir. Preguntas frecuentes sobre la ILE [monografía en internet]. Ciudad de México: ANDAR, 2010. [consultado 20II enero 18]. Disponible en: http://www.andar.org.mx/ minisitio\%20andar/preguntasfrecuentes.htm.

23. Rash V, Wielandt $H$, Knudsen L. Living conditions, contraceptive use and the choice of induced abortion among pregnant women in Denmark. Scand J Public Health 2002; 30:293-299.

24. Díaz-Díaz E, Álvarez-Vázques L, Farnot-Cardoso U. Factores de riesgo demográficos y sociales del aborto. Rev Cubana Salud Pública 200I;27(I):26-35.

25. Castañeda-Abascal I, Molina-Estévez M. Factores biosociales que influyen en la aparición del aborto provocado. Rev Cubana Obstet Ginecol 1999; 25(I):55-60.

26. Cabezas-García E, Langer-Glass A, Alvarez-Vázquez L, Bustamante $P$. Perfil sociodemográfico del aborto inducido. Salud Publica Mex 1998;40: 265-271.

27. Lie M, Robson S, May C. Experiences of abortion: a narrative review of qualitative studies. BMC Health Serv Res 2008:8: 150.

28. Grupo de Información en Reproducción Elegida. Cifras sobre el aborto en el DF 2007-20I I [monografía en internet]. Ciudad de México: GIRE, 20II [Consultado: 20II enero 6] Disponible en: http://www.gire. org.mx/contenido.php? informacion=222

29. Sousa A, Lozano R, Gakidou E. Exploring the determinants of unsafe abortion: improving the evidence base in Mexico. Health Policy Plan 2010; 25(4):I-II. 